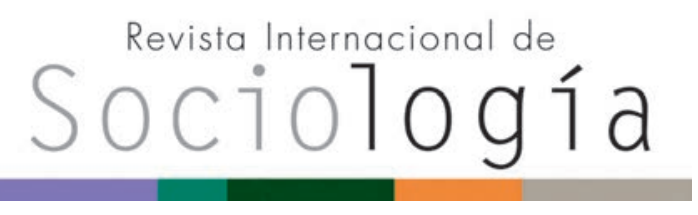

Revista Internacional de Sociología RIS

vol. 75 (3), e070, julio-septiembre, 2017, ISSN-L:0034-9712

doi: http://dx.doi.org/10.3989/ris.2017.75.3.15.189

\section{ALUMBRANDO LA MUERTE. Profesionales de la vida gestionando el duelo}

\author{
José Manuel Hernández Garre \\ Universidad Católica de Murcia, España. \\ jmhernandez@ucam.edu \\ ORCID iD: http://orcid.org/0000-0002-2507-3121
}

\author{
Francisca Carmen Sánchez Sánchez \\ Matrona del Hospital Los Arcos del Mar Menor, España. \\ paquita1974@gmail.com \\ ORCID iD: http://orcid.org/0000-0002-9391-8536
}

\author{
Paloma Echevarría Pérez \\ Universidad Católica de Murcia, España. \\ pechevarria@ucam.edu \\ ORCID iD: http://orcid.org/0000-0002-2458-611X
}

Cómo citar este artículo / Citation: Hernández Garre, J. M., F. C. Sánchez Sánchez y P. Echevarría Pérez. 2017. "Alumbrando la muerte. Profesionales de la vida gestionando el duelo". Revista Internacional de Sociología, 75 (3): e070. doi: http://dx.doi.org/10.3989/ris.2017.75.3.15.189

\section{RESUMEN}

El objetivo del artículo ha sido investigar las principales representaciones, experiencias y modelos de afrontamiento que desarrollan los profesionales sanitarios implicados en la atención al duelo perinatal. Se utilizó un enfoque cualitativo y fenomenológico realizando una serie de entrevistas semiestructuradas a profesionales de distintas categorías de las áreas obstétricas de tres hospitales públicos de la región de Murcia. Los relatos hablan de profesionales entrenados para la vida que han de enfrentarse a la muerte, de una falta de formación institucional ante la que los profesionales reaccionan recurriendo a la experiencia, la empatía o la formación autodidacta. Hablan de situaciones dolorosas que son vividas con tintes de tragedia, de cuidados dirigidos a la gestión psicológica del duelo, del tránsito desde modelos asistenciales de afrontamiento de los duelos basados en la evitación y el distanciamiento emocional hacia otros centrados en la verbalización de la experiencia y el contacto con el mortinato.

\section{Palabras Clave}

Duelo perinatal; Modelos de afrontamiento; Muerte neonatal; Profesionales sanitarios.

\section{GIVING BIRTH TO DEATH. Life professionals managing the bereavement}

Copyright: (C) 2017 CSIC. Este es un artículo de acceso abierto distribuido bajo los términos de la licencia Creative Commons Attribution (CC BY) España 3.0.

Recibido: 01/12/2015. Aceptado: 03/11/2016

\section{Abstract}

The aim of the study was to investigate the main representations, experiences and coping strategies developed by health professionals involved in perinatal bereavement care. A qualitative and phenomenological approach was used conducting a series of semistructured interviews to professionals of different categories of obstetric areas of three public hospitals in the region of Murcia. The stories talk about of professionals trained for the life they have to face death, talk about of a lack of institutional training to the professionals react drawing on the experience, empathy or self-taught. They talk about painful situations that are experienced by clinicians with hints of tragedy, speak of care directed to the psychological management of mourning, talks about the transition from coping models duels based on avoidance and emotional detachment to others centered on the verbalization of experience and contact with the stillborn.

\section{KeYWORDS}

Coping Models; Health Care Professional; Neonatal death; Perinatal Bereavement. 


\section{INTRODUCCIÓN}

La muerte es una realidad inesperada que deja cicatriz, que genera duelos, proyecciones de amor hacia quien desapareció. Se trata de una consecuencia del apego (Kübler-Ross 1969; Bowlby 1980), de una respuesta emotiva que se manifiesta con signos visibles externos, con comportamientos sociales y expresiones religiosas (Pangrazzi 1993). Quizás por ello no existe cultura "sin culto a los antepasados, sin ritualización del duelo, sin lugares y modos institucionales de sepultura" (Derrida 1996).

Estamos ante una situación compleja, difícil de explicar, cuyo abordaje es cuestión de debate en el marco de la teoría social. En este sentido, aún a costa de simplificar el fenómeno, se pueden señalar dos posturas antitéticas a la hora de afrontar el duelo y la muerte: las centradas en la expresión, en la verbalización y el contacto con directo con la muerte y el muerto, y las que se sitúan en el polo opuesto de la ocultación, de la negación y del alejamiento de la expiración y el difunto.

Los primeros postulados encuentran su estatus de posibilidad en las características del hombre tardo moderno, en el tiempo que los sociólogos han bautizado como segunda modernidad (Giddens 1993; Beck 1998), modernidad reflexiva (Giddens 1993; Beck 1998), contramodernidad (Beck 1998) o postmodernidad (Bauman 2001). Es decir, en un periodo caracterizado por la exigencia de enfrentar determinados riesgos, de estar en una constante evolución autorreflexiva que permita la construcción de la identidad. De un espacio en el que la inquietud, el dolor o la desesperación se entremezclan con la exacerbada seguridad en ciertas formas técnicas y sociales especializadas, en la confianza en nuevas terapéuticas y procesos de ayuda que promuevan que el sujeto sea un agente activo de su vida. En este habitus cobra sentido la propuesta de afrontamiento de la muerte que hace más de veinte años planteó Walter (1994), su proposición de un nuevo modelo de la muerte en el que la verbalización de la experiencia y la presencia de la terapia se convierten en elementos nucleares. Se trata de un sistema desde el que se proponen formas no negacionistas de hablar de la muerte, en las que esta está presente con toda su idiosincrasia en el debate social, en las que se puedan verbalizar libremente las emociones en el seno familiar sin que la pérdida se convierta en un tema tabú. Propuestas en sintonía con el modelo explicativo del duelo de Kübler-Ross (1969) y sus cinco etapas emocionales progresivas -negación, ira, negociación, depresión y aceptación-, en el sentido de que desde esta perspectiva el afrontamiento directo de la muerte, y la promoción de la verbalización de la experiencia y del contacto con el fallecido, ayudaría al afectado a no quedar encerrado en sí mismo, a salir del peligro de una perpetua fase de negación, a no caer en el horror de un duelo diferido.
En contraposición con estas posiciones se encuentran las actitudes caracterizadas por la ausencia de verbalización de la pérdida, por la no manifestación de síntomas de aflicción o trauma. Posicionamiento que parte de la constatación de una resistencia natural a la muerte que se instituye en componente principal de los procesos de duelo, se entiende pues que las personas no sepan qué hacer con los muertos, no sepan qué sentimientos deben tener o cómo expresarlos, ni que conducta seguir ante el fallecimiento (De Miguel 1995). Se trataría de posicionamientos más en línea con el modelo de afrontamiento del duelo propuesto por Bonanno (2009) en el que juega un papel central la resistencia natural a la muerte, cobrando sentido posturas ante el duelo en las que la no expresividad del dolor, incluso la risa y la sonrisa, pueden ser respuestas sanas del individuo reflejo de un mayor resiliencia ante la pérdida, y no siempre la expresión de una negación del proceso a la que haya que temer. Desde esta perspectiva se sostiene que no están suficientemente probadas las políticas públicas de afrontamiento emocional basadas en pedir a la gente que hable y llore la pérdida.

Dentro de este debate teorético sobre la muerte, un caso muy particular es el que acontece antes, durante o nada más nacer. Se trata de un tipo de muerte muy específico por "la proximidad entre el nacimiento y la muerte, por lo inesperado del proceso y porque los progenitores no han tenido la posibilidad de crear recuerdos significativos (Savage 1992; Stroebe y Schut 1999). Pero la muerte perinatal no solo afecta a los padres, sino también a los profesionales sanitarios que atienden el proceso (Nuzum, Meaney y O'Donoghue 2014). Se trata de expertos del área obstétrica, es decir de profesionales de vida, acostumbrados a gestionar el nacimiento y la alegría pero con limitaciones para gestionar la muerte perinatal (Wallbank y Robertson 2013). En esta línea, los estudios muestran como los especialistas están sometidos a una gran carga emocional experimentando dolor, desasosiego, ansiedad, frustración, ira, tristeza, pena, culpa, inseguridad, estrés o impotencia (Gardner 1999; Gálvez 2006; Nuzum, Meaney y O’Donoghue 2014; Jones y Smythe 2015). Los técnicos manifiestan una falta de información específica para afrontar estas situaciones (Gardner 1999; Gálvez 2006; Pastor et al. 2011; Jones y Smythe 2015), haciendo hincapié en la necesidad de una mayor educación y preparación para el correcto afrontamiento de la muerte antes, durante, o nada más nacer (Allchin 2006). La mayoría de autores coinciden, también, en señalar que las emociones (García Caro et al. 2010) o las creencias de los profesionales (Cacciatore 2010) determinan su asistencia (Gold, Dalton y Schwenk 2007) por encima de otros factores como los propios deseos de los padres o los diferentes modelos de afrontamiento propugnados por la teoría social (Cacciatore 2010). En este contexto, según las investigaciones previas, las conductas 
evitativas de huida, olvido o distanciamiento (JonasSimpson et al. 2010; Wallbank y Robertson 2013; Puia, Lewis y Beck 2013) forman parte integral del itinerario asistencial al duelo perinatal; centrándose los profesionales en los cuidados físicos, a la vez que evaden otras demandas emocionales de los padres mediante expresiones de invalidación, desautorización, minimización, rechazo, impaciencia o desinterés, en lo que parece un intento por gestionar su propio impacto emocional (Gálvez 2006; Fenwick et al. 2007; Wallbank y Robertson 2013; Nuzum, Meaney y O’Donoghue 2014). Desde esta premisa, y a pesar de que se trata de un fenómeno con cierta relevancia epidemiológica ${ }^{1}$, parece que estamos ante duelos desautorizados institucional y socialmente (Doka 2002), ante actuaciones profesionales basadas en la negación, en el silencio, en la ocultación del neonato a los ojos de los padres, en el intento de minusvalorar el proceso en aras de posibles maternidades venideras (Bourne 1968). A todo ello se suma el hecho de que no parece existir un sistema de apoyo dentro del mismo centro sanitario o entre los propios compañeros que ayude a gestionar el impacto emocional del proceso (Gálvez 2006; Fenwick et al. 2007; Chan et al. 2008; Wallbank y Robertson 2013; Nuzum, Meaney y O’Donoghue 2014).

Casi la totalidad de estudios parte de posicionamientos teóricos en línea con el modelo explicativo del duelo Kübler-Ross, y las estrategias de afrontamiento propugnadas por Walter, en las que la expresividad de las emociones, la terapia y el contacto con la muerte y el muerto son eje central. Desde esta atalaya los estudios apuestan por fomentar la participación de los padres en la toma de decisiones (Gálvez 2006; Côte-Arsenault y Denney-Koelsch 2011), insistiendo en la necesidad de implantar programas de formación y guías de práctica clínica que ayuden a los profesionales a adquirir los conocimientos y capacidades en el manejo del duelo perinatal. Se parte de la conveniencia de fomentar la expresividad de las emociones por parte de los padres (Cacciatore 2010; 2013), desarrollando estrategias como la investigación en acción participativa (Pastor et al. 2012) que inserte a los padres en modos de acción definidos que los ayuden a hacer un afrontamiento de no negación del dolor (Jonas-Simpson et al. 2013). Concordato solo roto por alguna publicación minoritaria en la que se cuestiona la pertinencia de alentar en los padres el contacto con su niño muerto, apuntando que no existe suficiente evidencia sobre el tema y que este contacto podría inducir a un trastorno de estrés postraumático (Badenhorst y Hughes 2007).

Estamos pues ante una situación paradójica en la que, si bien la mayoría de publicaciones parte de modelos de afrontamiento en pro de la expresividad y el contacto con la muerte, a su vez muestran una realidad clínica antagónica marcada por la evitación, la negación, el silencio, la ocultación del neonato y el intento de minusvalorar el proceso. Ante esta realidad se plantea la necesidad de desarrollar futuras investigaciones que monitoricen el estado actual de la cuestión y desarrollen posibles estrategias positivas (Fernández et al. 2012). Contexto en el que se plantea el presente estudio, cuyo objetivo ha sido investigar las principales representaciones, vivencias y estrategias de afrontamiento que manejan los profesionales sanitarios implicados en la atención al duelo perinatal. Los análisis se han realizado desde un posicionamiento analítico que busca comprender cuales son los modelos sociológicos de afrontamiento del duelo perinatal hegemónicos entre los profesionales implicados en el proceso. Se trata, en definitiva, de investigar si persisten todavía los itinerarios asistenciales marcados por la negación y el distanciamiento profesional, como parecen apuntar las investigaciones anteriores, o por el contrario se han introducido otros modelos basados en la verbalización de la experiencia y el contacto con la muerte. Modelos ya introducidos por Walter hace más de veinte años y adoptados por la mayoría de la literatura actual sobre el duelo perinatal como los deseables.

\section{Metodología}

La naturaleza del fenómeno de estudio -la pérdida, la muerte, el duelo- determinó la elección de un diseño de carácter cualitativo que desde un enfoque fenomenológico recogiera las principales representaciones, vivencias y estrategias asistenciales de los profesionales sanitarios implicados en los procesos de atención del duelo perinatal. El duelo como objeto de investigación se sitúa en el ámbito emocional, está íntimamente unido a lo anímico, al sentimiento; por lo tanto su naturaleza es eminentemente subjetiva, heterogénea, personal (Gálvez 2006). En este sentido, el enfoque fenomenológico permite construir la realidad tal y como la observan los actores (Hernández, Fernández y Batista 2014), tratándose además de un método de investigación cada vez más utilizado en el campo sanitario, al enriquecer sobremanera las perspectivas epidemiológicas.

El estudio se llevó a cabo en tres hospitales de la red pública de la región de Murcia: Hospital Universitario Rafael Méndez (Lorca), Hospital de los Arcos del Mar Menor (San Javier) y Hospital Virgen del Castillo (Yecla). Se trata de centros comarcales de tamaño intermedio que dan cobertura a las Áreas III, VIII y $\mathrm{V}$ respectivamente, y con un volumen de partos al año que oscila entre los 2000 partos del Hospital Rafael Méndez, los 1400 del Hospital los Arcos del Mar Menor y los 800 del Hospital Virgen del Castillo. La elección de los centros vino determinada por la facilidad de acceso al trabajo de campo en dichas instituciones, ya que estas mostraron su interés por conocer los posibles déficits asistenciales en aras de una mejora asistencial. La investigación se centró en las áreas de paritorio de los tres centros, debido a la 
necesidad de seleccionar un ámbito clínico en el que los profesionales tuvieran un contacto significativo y directo con la muerte perinatal. En este sentido el paritorio es el contexto idóneo, ya que es precisamente durante el parto donde la muerte del feto se hace más palpable y dolorosa, donde lo inesperado y la proximidad entre el nacimiento y la muerte marca la diferencia (Mander 1994), siendo por ello los profesionales de este área los más implicados en el fenómeno de estudio.

La información se obtuvo a través de la realización de una serie de entrevistas semiestructuradas al personal sanitario de los servicios de paritorio de los tres centros señalados que habían tenido contacto asistencial con situaciones de muerte y duelo perinatal. La técnica de investigación se seleccionó bajo la premisa de que las entrevistas recogen las vivencias y perspectivas de los implicados en las situaciones de estudio desde sus propias palabras (Taylor y Bogdan 1998). En todo momento se persiguió una implicación que facilitara la recogida e interpretación de los datos, permitiendo adentrarse mejor en su significado, sentido y comprensión (Gálvez 2006). Se buscó, en definitiva, un conocimiento interno del escenario y del trasfondo escénico (Goffman1987), desde el que se pudiera interpretar y ver el mundo de manera similar a los informantes (Hammersley y Atkinson 1994).

Las entrevistas se realizaron entre los meses de marzo y julio del año dos mil quince, de forma individualizada y en la sala de descanso del personal aprovechando los descansos durante su jornada laboral. Tras las autorizaciones pertinentes de los diferentes centros clínicos, y una vez recogidos ciertos datos demográficos como la edad, el sexo, la profesión y los años de experiencia, se procedió a su realización desde un enfoque abierto, intentando recoger lo que ocurría, lo que los entrevistados pensaban que ocurría y lo que creían que debería ocurrir. En todo momento se intentó crear un ambiente de cordialidad que propiciara una relación empática con los entrevistados (Taylor y Bogdan 1998), introduciendo de manera flexible una serie de temas a debatir, en base a las categorías de análisis, con el objetivo de facilitar la fluidez del discurso de los informantes. El guion de las mismas giró en torno a tres puntos principales: la formación, la gestión de emociones y las estrategias asistenciales de afrontamiento del duelo desplegadas. Antes de la realización de las mismas se le facilitó información escrita sobre el objetivo y procedimiento del estudio, obteniéndose la autorización y un consentimiento informado de los participantes. En todo momento se respetó la confidencialidad de los testimonios en base a la Ley Orgánica 15/1999 de 13 de diciembre de Protección de Datos de Carácter Personal (LOPD). El anonimato de los participantes se aseguró mediante la asignación de una clave numérica a cada entrevista recuperando a posteriori los datos en base a las categorías de análisis (Coffey y Atkinson 2005).

La selección de la muestra se realizó mediante un muestreo intencional con el objetivo de garantizar que los participantes fueran informantes clave. Asimismo se siguió el criterio de proximidad, intersubjetividad y saturación para garantizar una mayor representatividad y validez interna de la muestra, homogeneizándola en base a los siguientes criterios de inclusión:

- Ser trabajador de los servicios de paritorio de los tres centros seleccionados con más de un año de experiencia.

- Prestar una atención directa a las embarazadas/parturientas.

- Haber atendido en el último año al menos un caso de muerte perinatal.

- Dar el consentimiento para colaborar en la investigación.

La utilización de estos criterios de inclusión vino dada por la necesidad de delimitar claramente el perfil relevante de la población susceptible de participar, asegurándonos la inclusión de profesionales sanitarios que hubieran prestado atención directa y significativa en los procesos de muerte perinatal. En este sentido, y como ya se ha comentado, los trabajadores de paritorio, obstetras, matronas y auxiliares, son los que prestan atención directa a este colectivo, sufriendo de primera mano los efectos psicológicos del mortinato (Bourne 1968).

Para determinar el tamaño de la muestra se siguió el criterio de saturación, considerando que esta se produce cuando la información obtenida es repetitiva y no aporta nuevos datos significativos a la investigación (Bertaux 1993). Siguiendo este criterio la información se saturó al llegar a treinta y siete informantes, cinco obstetras, veinticuatro matronas y ocho auxiliares de enfermería. De ellos treinta y cinco eran mujeres y dos hombres (los dos, ginecólogos) con edades comprendidas entre los veintiocho y los sesenta y un años, y en todos los casos tenían más de tres años de experiencia. La preeminencia de matronas en la muestra vino determinada por la importancia de su relato, ya que son las encargadas de estar a pie de cama con los implicados, gestionando en primera persona durante todo el trance los procesos de duelo, por lo que sus informaciones resultan doblemente significativas.

El análisis se basó en la teoría fundamentada (Grounded Theory) estableciendo un orden en la codificación del discurso: codificación abierta e identificación de categorías, codificación axial y relación de categorías y codificación selectiva y refinamiento de las categorías centrales (Strauss y Corbin 1998). Las categorías se describieron individual y relacionalmente (Glaser y Strauss 1967; Charmaz 2006) a través de una comparación constante, realizando 
a posteriori un muestreo teórico que permitiera seleccionar aquellos testimonios que resultaban más apropiados para desarrollar y probar las ideas analíticas emergentes (Strauss y Corbin 1990). Las categorías de análisis surgieron de la exhaustiva revisión bibliográfica y del análisis del discurso, estableciéndose finalmente tres categorías principales:

- Los conocimientos y formación que manejan los profesionales sobre el duelo perinatal.

- Las emociones y gestión de sentimientos que realizan los asistentes.

- Y el tipo de cuidados, estrategias y modelos de afrontamiento que despliegan los sanitarios.

Se pretendió recoger información estratégica sobre la percepción de los propios profesionales con el fin de construir las visiones, creencias y mecanismos institucionales que hay detrás de los procesos asistenciales del duelo perinatal. El análisis se realizó desde un posicionamiento analítico que buscaba comprender qué modelo sociológico de afrontamiento del duelo perinatal es actualmente el hegemónico entre los profesionales que atienden al duelo perinatal, intentando constatar si predominan los afrontamientos de evitación o los que fomentan la expresión de emociones y el contacto con el mortinato. Más allá de las limitaciones propias del enfoque fenomenológico pensamos que se ha recogido información relevante que refleja las cosmovisiones más significativas sobre duelo perinatal que manejan los profesionales sanitarios.

\section{Resultados / Discusión}

Los resultados surgen de las tres categorías emergentes del discurso de los profesionales: el grado de conocimiento, la gestión de las propias emociones, y los cuidados y modelos de afrontamiento desplegados. Hilo conductor que vertebra los relatos de los clínicos sirviendo a su vez de guía en la exposición de los mismos.

\section{De informaciones y desinformaciones. En busca de 'El dorado' del conocimiento}

En concordancia con la mayoría de investigaciones previas los discursos hablan de paradojas, de vacíos inexplicables, de dificultades para afrontar la perdida y de falta de formación institucional. Testimonian la complejidad de la muerte, el temor ante la idea de una total extinción que rebasa el entendimiento humano. Surgen, de esta manera, en el entramado discursivo ciertos "tic", ciertos códigos homogéneos sobre la complicación que supone afrontar una realidad clínica que se percibe con tintes de irrealidad (Wallbank y Robertson 2013): "Nunca se está preparado para esos momentos, son momentos de irrealidad que muchas veces no queremos aceptar como profesionales, el duelo de un nonato es algo difícil de asimilar" (E37G) ${ }^{2}$. De un trance inconmensurable y de difícil abordaje: "Creo que profesionalmente nadie está capacitado para atender el duelo de estos pacientes. La paciente no está preparada para este imprevisto y la mayoría de las veces el profesional de paritorio tampoco. Son situaciones difíciles" (E16G). Se trata de una cuestión fronteriza, de una vivencia que pone a prueba los recursos para vencer el miedo al dolor del otro, para asimilar la vulgaridad de la propia vida (Gabilondo 2003).

Sin embargo, y pese a complejidad del duelo perinatal, los diferentes perfiles profesionales coinciden en señalar una falta de formación institucional: "No existe formación dirigida a profesionales en el que se nos informe sobre los aspectos a considerar, lo que debemos decir en esos momentos, grupos de apoyo" (E36M); "Yo personalmente no he recibido ninguna formación" (E7A). Al trasluz de los testimonios se deja ver la contradicción entre la dificultad del proceso y la falta de información operativa (Pastor et al. 2011; Jones y Smythe 2015): "como enfermera me hablaron sobre las fases del duelo, pero a la hora de ponerlas en práctica en una situación real, carezco de una estrategia de abordaje" (E23M); "estoy capacitada regular porque al no tener información no sé cómo actuar, intento hacer lo mejor que sé" (E32A). En este sentido, el área de seguridad de los informantes parece estar en los cuidados biológicos, en el tratamiento de las dimensiones físicas, quedando en el ángulo oscuro del aprendizaje los aspectos psicológicos y sociogrupales (Gardner 1999; Gálvez 2006): "No he recibido formación específica. A nivel biológico tengo suficientes conocimientos, pero a nivel psicológico y socio familiar no" (E16G); "En la esfera de lo biológico estoy más capacitada porque la docencia sanitaria está más centrada en la formación en patología física dejando en plano secundario los aspectos psicológicos o socio familiares" (E36M). Surge aquí el biologicismo que impregna los itinerarios asistenciales, la desafección clínica hacia las esferas psicosociales de la atención (Wallbank y Robertson 2013; Nuzum, Meaney y O’Donoghue 2014): “Me faltan conocimientos en este campo sobre todo en lo relativo al apoyo psicológico" (E1M); "Necesitaríamos formarnos periódicamente para tener más herramientas a la hora de afrontar este trance sobre todo a nivel psicológico y socio familiar" (E9M). Las lagunas parecen estar, pues, en el abordaje emocional de la pérdida, en una gestión psicológica del duelo (Gálvez 2006; Fenwick et al. 2007): "En realidad no sabría diferenciar mis cuidados de feto cruz ${ }^{3}$ de los de un feto vivo" (E25M).

Ante el déficit institucionalizado de un proyecto formativo serio en estas materias, los profesionales desarrollan estrategias muy variopintas de aprendizaje. En este sentido, están los que se nutren de su experiencia profesional o de la de sus compañeros: 
"Formación como tal no, se aprende de la experiencia de otro" (E37G); "Mis conocimientos vienen de mi experiencia profesional y del sentido común" (E2M); "No he recibido formación por desgracia he aprendido con los casos que me ha tocado asistir" (E28A). También los hay que tiran de sus experiencias personales: "No he recibido información directa y específica sobre el duelo pero he vivido de cerca el proceso de duelo de mi hermano que abortó hace un año a los 5 meses de gestación" (E31A). Otros emprenden un proceso formativo autodidacta: "He recibido formación [...] por mi cuenta, en libros de ginecología y en un máster" (E30G); "He realizado una formación autodidacta, ya que me he visto en la obligación de formarme por la impotencia que he sentido al no encontrarme con recursos para hacer frente a este tipo de problemas" (E2OM). También los hay que han realizado cursos al margen del itinerario académico oficial: "En la formación básica y residencia, no tuve ningún tipo de formación, solo nociones generales del duelo pero he asistido a jornadas sobre duelo perinatal impartidas por profesionales colegas" (E9M). Entre los que sí recibieron algún tipo de formación durante su formación curricular, surge una constante en su discurso, fue escasa e insuficiente: "Recibí un seminario de una hora en mi residencia. Las unidades docentes dedican tiempo escaso o incluso nulo y tampoco existen cursos de formación continuada que traten el tema“ (E36M)). Por último, están aquellos que no recibieron ningún tipo de formación que tienen que recurrir a su capacidad creativa, a sus aptitudes empáticas o simplemente se dejan llevar por sus emociones (García-Caro et al. 2010): "No tengo conocimientos suficientes salvo creatividad y empatía, salvo que en mi forma de ser he desarrollado capacidades con el contacto con la paciente" (E22M); "trato de echar trato de la empatía [...] sobre todo a nivel psicológico y socio familiar" (E29A). Parece que estamos, en cierta medida, ante duelos olvidados (Kirkley-Best y Kellner 1982) por lo menos durante el proyecto curricular de los profesionales.

Esta falta de un programa homogéneo de formación no es baladí, se traduce en dudas profesionales, en la frustración de no saber si se está haciendo lo correcto: "Me preocupa saber actuar profesionalmente porque estos casos tiene sobreañadido una serie de complicaciones que pueden afectar negativamente a la salud de la madre" (E25M); "cuando se han dado estos casos intentas ayudar en la medida de lo posible al no haber recibido formación en este campo no sé si la ayuda ha sido la acertada" (E17A). Queda el rastro de la duda, la ansiedad sobre si las actuaciones no habrán sido incluso perjudiciales: "muchas veces actuamos o decimos cosas pensando que ayudamos y justo conseguimos todo lo contrario" (E27M). En este sentido, se deja sentir la falta de una verdadera política institucional de gestión de situaciones de dolor en obstetricia (Elia 1959).
En este habitus de la desinformación los testimonios coinciden en la importancia de una mayor implicación institucional que posibilite un mayor capacitación profesional en la materia (Gálvez 2006; Fenwick et al. 2007; Chan et al. 2008; Wallbank y Robertson 2013; Nuzum, Meaney y O'Donoghue 2014): "Este tema debe ser tratado por el sistema sanitario de forma más profunda, instaurando protocolos de actuación y formación obligatoria de todos los profesionales" (E8M). Destaca la importancia de una mayor información y formación continuada (Roehrs et al. 2008) ante la realidad de una muerte olvidada que, sin embargo, todos los sanitarios identifican como inevitable (Delgado y Monzón 2002): "Debería de darse formación específica sobre duelo antenatal a los profesionales implicados en el trabajo obstétrico" (E37G); "Es obligatorio que todos los profesionales de salud implicados en esta materia reciban formación específica sobre el abordaje de este tema"(E35M); "Se debe de incluir de forma más exhaustiva el tema del duelo perinatal en el grado y especialidad sobre todo, así como en la formación continuada y de postgrado, ya que se observa un déficit importante de oferta formativa en este“ (E20M). En este sentido, los discursos contraponen la situación de sus centros con los abordajes de otros países: "En otros países atienden y animan a estas parejas. Se pone una señal en la puerta de su habitación y toda la institución sabe cómo actuar. En mi hospital es un tema poco abordado" (E26M). Y eso que no parece un tema menor, sobre todo si tenemos en cuenta que la formación mejora, según las evidencias, las actitudes positivas ante la muerte perinatal (Chan et al. 2008), permitiendo afrontar con más confianza y sensibilidad cada pérdida (Parkes 1972).

\section{De sentimientos y emociones. Autonegociar la muerte en el "Shangri-La» de la vida}

En línea con la mayoría de estudios previos, los relatos hablan de la paradoja de unos profesionales entrenados para la vida que no se encuentran preparados para gestionar la muerte (Wallbank y Robertson 2013): "No estoy acostumbrado a tratar de forma cercana con la muerte por mi especialidad" (E16G); "La muerte es una situación poco habitual y me crea un estado muy diferente en actitudes y sentimientos que cuando se trata de un bebé con vida" (E27M). Surge la contradicción de una situación normalmente ligada a la felicidad que se presenta impregnada del sufrimiento: "Asocias el nacimiento a felicidad y cuando nace un feto cruz, nuestros sentimientos pasan a ser totalmente negativos" (E37A); "Estamos acostumbrados a que sea un acontecimiento feliz y en este caso me es difícil animar a la paciente" (E30G). Nacimiento y muerte se entrelazan en una combinación inusual, ilógica, que es difícil de negociar: "Es muy traumático pasar por un parto cuando sabes que el final no es el 
resultado de un bebé lloroso y activo, sino todo lo contrario, es un silencio aterrador" (E14A). Parece que es el precio emocional que hay que pagar por una situación que se "diferencia de otros duelos por la proximidad entre el nacimiento y la muerte, por lo inesperado de un proceso". (Mander 1994:85) en el que se muere antes de nacer.

No ha de extrañar, pues, que los expertos lo vivan como una situación con tintes de tragedia, como una realidad traumática que perturba profundamente su esfera anímica: "Suelo quedar muy afectado y luego uno tiene que seguir atendiendo a otros pacientes como si nada hubiera pasado y no es así" (E16G); "A veces el sentimiento de tristeza me dura uno o dos días afectando a mi vida personal"(E18M); "Es difícil olvidarlo en varios días e incluso se mezclan estas situaciones vividas en tus sueños" (E14A). Se trata de pulsiones vegetativas, a veces incluso somatizadas (Heazell et al. 2012): "Empecé a sentir entumecimiento. Sentí frío y calor al mismo tiempo. Ya no pude trabajar el resto del día" (E10M). De vivencias próximas al estrés postraumático, de desventuras que prevalecen en la memoria: "Son casos que nunca se olvidan, siempre se recuerdan a la mujer y a la familia como si fuera ayer, son casos que se llevan a casa" (E19M); "Esas vivencias, esas sensaciones te acompañan ya siempre en tu vida porque fueron vividas con una gran carga emociona" (E14A). Experiencias que incluso en algunos profesionales precisan para su gestión del recurso a lo espiritual y religioso: "Es un gran consuelo apoyarte en tus creencias espirituales para poder aceptar estas experiencias" (E14A); "La fe, si eres creyente, te da gran entereza para comprender este tipo de situaciones tan extremas." (E14A); "Si tienes buena base religiosa el momento se vive con más serenidad" (E14A). Parece que se trata de una herramienta que da sentido a la muerte (Marín 2007), y en cierta medida, a la vida, ya que todo intento de encontrarle sentido a esta recae en una reflexión sobre la propia muerte (Camus 1965).

En este contexto la batalla se juega en el terreno sensitivo, en la autogestión de emociones, al enfrentarse a una enemiga que es la más temida de las separaciones (Pangrazzi 1993). Surge, de esta manera, en los profesionales toda una cascada emocional que abarca todo el arco iris de lo sensitivo: impotencia, incertidumbre, miedo, dolor, tristeza, desolación, ira, culpabilidad, negación, evitación, rechazo, empatía, paternalismo, ganas de llorar (Worden, Aparicio y Barberán 1997; Jones y Smythe 2015). Así, ante lo inevitable e irreversible del proceso germina la semilla de la frustración (Nuzum, Meaney y O’Donoghue 2014): "Siento sensación de impotencia y frustración por el tremendo golpe" (E5G); "Lo primero que me surge es impotencia por saber que no se puede hacer nada por el feto que acaba de fallecer". (E24M); "Impotencia ante la des- esperación de los padres que no tienen consuelo porque sus ilusiones se desmoronan en segundos" (E28A). Florece el miedo a morir, a los muertos, a lo que acontece tras la misma, tapizando el discurso de los profesionales de incertidumbre e inseguridad (Gálvez 2006; Marín 2007): "Preocupación e incertidumbre por cómo se va a desarrollar el parto y por las emociones y sentimientos que surgirán" (E24M). "Me aflora sobre todo miedo [...] me producen rechazo por el propio miedo de aceptar la muerte de un bebé cuando cronológicamente no estamos preparados"(E10M); Nace "un dolor sombrío, subyacente a los sentimientos“ (Mejías, García y Duarte 2012: 105), no tanto por lo que ha sido sino por lo que pudo haber sido (Savage 1992): "Siento dolor por los progenitores y me afecta personalmente [...] siempre llevo en mi mente un caso así" (E25M); "Sentía dolor, tristeza y desolación de ponerme en el lugar de la persona afectada, me lo llevaba al ámbito personal sin saber separar la frontera.(E6M).

Se trata de un dolor único, individualizado (Morin 1994), de una profunda sensación de desolación y tristeza ante unos padres que ni siquiera han visto al hijo, ni lo han tenido en brazos (Gardner 1999; Savage 1992): "Fundamentalmente siento tristeza, el parto es el momento de la mujer en que se espera la llegada de la vida, nunca se está profesionalmente lo suficientemente preparada para recibir la llegada de la no vida" (E36M); "Tristeza, desolación y angustia ante la pérdida de un ser querido tan esperado por los padres" (E6A). Ante la imposibilidad de contacto, de crear recuerdos (Stroebe y Schut 1999), de alcanzar "realidades soñadas, deseadas y jamás alcanzadas" (Pangrazzi 1993;18), brota la ira, la culpa, la sensación de fracaso: "Sentimiento de [...] de fracaso por buscar la causa e intentar encontrar una causa médica del óbito" (E30G); "Se siente ira [...] sentimientos ambiguos con polos opuestos" (E19M); "Sentimientos de culpabilidad y dolor por la pérdida y la situación socio-familiar" (E22M). Fluyen las emociones, la tristeza e incluso el llanto contenido (Puia, Lewis y Beck 2013): "Estaba intentando contener mis lágrimas, solo pensaba en la pena que iba a sentir la pobre mujer, tras el diagnóstico fueron momentos muy duros y ya no pude contenerlas" (E1M); "Hay momentos que tengo que salir de la habitación para evitar sacar mucho a la luz mis emociones," (E2M). Son experiencias estresantes que necesitan ser verbalizadas para aliviar la carga emocional: "Atender un parto de estas características siempre afecta personalmente, intento comentarlo y hablarlo con otros compañeros para reducir este grado de afectación"(E36M).

El dolor y la impotencia se transforman en algunos casos en negación y rechazo a la muerte (JonasSimpson et al. 2010): "El primer sentimiento es del rechazo a la muerte, intento evitar la situación, preferiría no atender a la paciente," (E18M);"Mi dificultad asis- 
tencial es el rechazo a la muerte pues me afecta profundamente así como la espiral de sentimientos negativos a los que me voy a someter en el transcurso de esta situación" (E24M). Ante la dificultad de gestionar una situación fronteriza como la pérdida aflora la evitación (Marín 2007; Nuzum, Meaney y O’Donoghue 2014), la huida (Gálvez 2006), el olvido (Wallbank y Robertson 2013), emerge el silencio (Bourne 1968), la realidad de unos duelos que parecen desautorizados (Doka 2002): "Espero asistir los menos partos de feto cruz posible en los años profesionales que me quedan" (E14A); "Por lo general, los que atendemos a un parto de este tipo estamos un poco impactados [...] Si pudiéramos elegir, creo que lo evitaríamos" (E2M). Son situaciones inverosímiles, y las reacciones oscilan desde la coraza que protege de lo inabarcable de la muerte (Morin 1994; Puia, Lewis y Beck 2013): "Con el tiempo he ido evitando la implicación emocional, creado una pequeña coraza y abordándolo con la mayor asepsia posible" (E23M); "Es duro enfrentarse a los sentimientos que provoca en una misma, dolor por la pérdida ajena, una profunda tristeza y rabia porque tengan que pasar cosas asi" (E12M). En el otro extremo surge el proteccionismo y el paternalismo: "Te identificas con la paciente, te pones en su lugar [...] te cuesta mantener la distancia ante un suceso traumático"(E30G); "En todo el proceso sé que reflejo proteccionismo, esto no sé si es bueno o por el contrario perjudica el proceso de afrontamiento que ha de hacer la mujer." (E23M); "Realmente empatizo, es una situación de gran sufrimiento para unos padres y familia y como en cualquier pérdida siento tristeza por ellos" (E26M). El hombre es para la muerte (Heidegger 1997), y la vivencia de la pérdida del otro toca sus pilares existenciales.

La muerte perinatal sigue habitando el terreno de lo ajeno, sigue siendo minimizada, negada en cierta medida (Fenwick et al. 2007; Payás 2010), y a pesar de que no hay lugar sin modos institucionales de sepultura (Derrida 1996) sigue estando impregnada de cierta negación y tabú: "El duelo perinatal es un tema tabú que no se suele tratar abiertamente" (E3M); "no estamos preparados para la muerte y seguimos viéndola como un tema tabú" (E37A). La muerte es algo biológico, pero también simbólico, cultural (Morin 1994), que en nuestra sociedad habita bajo el paraguas de la negación aunque según los testimonios ello suponga duelos patológicos: "Todo lo que tenga que ver con el proceso de la muerte en nuestra sociedad es vetado y censurado por el horror que nos supone el concepto de la muerte, el dolor y la enfermedad." (E20M); "Vivimos en una sociedad de espaldas al dolor, a la muerte e intentamos negar su existencia ignorando como elaborarla, creando a veces duelos patológicos que pueden interferir en la posterior vida de la pareja y la relación con sus hijos previos o futuros" (E9M). Desde esta representación en términos de tabú, los expertos no se sienten nunca totalmente preparados para afrontarla, para ne- gociar el duelo de sus pacientes: "El rechazo al sufrimiento provoca una torpeza generalizada a la hora de abordar la muerte, sobre todo en gremios profesionales como ginecólogos y algunas comadronas, [...] ello se traduce en una actitud frívola o generando estereotipos sobre la muerte como la insensibilidad o indiferencia afectiva, actitudes que son mecanismos de defensa inadecuados" (E20M). En este sentido, quizás sea el momento de devolver el sentido a la muerte, de rehumanizarla, de apostar por el futuro (Gómez 2003).

\section{De cuidados y modelos de afrontamiento del duelo. Gestionar la muerte nada más nacer}

A pesar de las dificultades para formarse, de la difícil gestión emocional y del tabú hacia la muerte, los testimonios muestran todo un abanico de cuidados, de tránsitos de despedida que se contraponen a los modelos de afrontamiento basados en la negación, la huida y el distanciamiento; afrontamientos percibidos por la mayoría de sanitarios como anacrónicas, como parte del pasado: "Hace muchos años todo este proceso mamá-feto cruz, no se realizaba, al bebé se le apartaba de la madre y se la consolaba diciéndole no pasa nada, eres joven tienes toda la vida por delate, la naturaleza es muy sabia. Hoy día todo ha cambiado y hay grupos de apoyo, psicólogos y los padres están rodeados de familiares y amigos" (E7A). En este sentido, nuestros resultados divergen de la mayoría de investigaciones previas que situaban el distanciamiento, la evitación o el rechazo de los profesionales como sus posicionamientos más habituales (Jonas-Simpson et al. 2010; Wallbank y Robertson 2013; Nuzum, Meaney y O’Donoghue 2014); a diferencia de las evidencias previas la mayoría de profesionales entrevistados muestran sensibilidad y preocupación por los padres y por la falta de protocolos específicos de atención: "Siento que la institución no cuida a estas familias [...] Odio la frase no te preocupes ya tendrás más, eres muy joven" (E26M); "En mi hospital no hay protocolo ante estas situaciones" (E8M). Lejos de la negación, la mayoría de testimonios apuestan por un modelo de gestión del duelo acorde con las propuestas de Walter (1994) en las que la verbalización de la experiencia, la presencia de la terapia y el contacto con el mortinato sean ejes centrales de la atención. En esta línea cobran peso en el discurso factores como el cuidado del entorno, el apoyo externo, la evitación del dolor, y sobre todo una gestión psicológica de la pérdida asentada en la actitud de respeto, el fomento de la despedida, la expresión de los sentimientos y el derecho a la información.

Cuidar el espacio físico y el entorno se convierte en elemento clave del discurso; en este sentido el silencio, la luz y la intimidad se instituyen en piedras angulares de la gestión ambiental: "En este caso cuidar especialmente el entorno, evitar ruidos, luces en 
exceso, paso innecesario de personal por la sala de dilatación o parto. [...] Mantenerlos lo más alejados de sonidos de monitores" (E23M); Se procura que sea lo más privado posible sin gente circulando, con el personal estrictamente necesario, que sea lo más tranquilo posible dándole tiempo para que pueda seguir adelante"(E13M). Otro aspecto que resalta en los discursos es la gestión selectiva de la habitación, procurando alejarlas de llantos, de compartir espacios con otras mujeres con bebés sanos: "Que no estén en la misma habitación con otras mujeres con bebés sanos" (E34M); "A veces se ingresa en otra planta para que no oiga los llantos de otro bebé“ (E28A); "Intentar buscar una habitación para su estancia en una sala distinta a la maternidad" (E35M). En definitiva, fomentar la serenidad, disminuir el ambiente traumático: "Intento aportar serenidad $y$ no crear un ambiente aún más trágico“ (E34M). En elemento central de la asistencia se convierte también el fomento del acompañamiento, de los apoyos externos durante el proceso: "Si para cualquier tipo de parto considero importante el acompañamiento, más todavía para estos casos en los que la madre necesita sentirse arropada y consolada" (E2M); "La mujer tiene que estar acompañada por su pareja o quien decida, dejarles espacio y respetar su intimidad y solo hacer acto de presencia si ella lo requiere" (E13M). En este marco surge en los discursos la necesidad de un apoyo familiar extrahospitalario, de recurrir a los grupos de autoayuda: "La paciente, una vez abandona el hospital debe seguir recibiendo ayuda tanto de su familia, como profesionalmente, si así fuese el caso. [...] Ahí es donde la familia tiene que ayudar, para que no se sume en su dolor $y$ pueda salir poco a poco de ese pozo en el que se encuentra". (E17A); "Son importantes los grupos de apoyo" (E7A). Se trata de un recurso imprescindible, ya que el apoyo hace más soportable la pérdida (Gálvez 2006). En cuanto a los cuidados físicos resalta una correcta gestión del dolor que no agrave el sufrimiento de la pérdida, que garantice que el peso de la carga será calmado, aliviado en la medida de lo posible (Pellegrino 1985): "Es fundamental para mí que la mujer no sufra dolor, le ofrezco lo antes posible la epidural" (E3M);"Intentamos minimizar al máximo el dolor, mórficos, epidural temprana, etc“ (E13M); "Para mí es importante que después de lo que los padres están sufriendo que la madre no sufra en ningún momento ningún dolor a la hora de parir" (E28A). Parece un elemento importante, ya que la pérdida, la muerte digna, se asocia en cierta medida con la gestión del dolor, con evitar el exceso de intervencionismo exterior (Domínguez y Urraca 1985).

Pero quizás sea en el abordaje psicológico donde se juegue la auténtica batalla, en este sentido, como se ha comentado, parece que se está asentando poco a poco un modelo de afrontamiento que busca la aceptación, el abandono del tabú de la muerte. Coordenadas desde las que el respeto de los sen- timientos ajenos cobra especial relevancia: "Hay que mostrar apoyo y respeto, procurar no incurrir en estereotipos y torpezas, ni contradecir ni discutir con ella" (E20M); "Lo primero es respetar su duelo e intimidad durante los primeros momentos" (E6A). Se trata de premisas desde las que la autonomía y el acatamiento de las decisiones de los padres se convierten en elementos capitales: "Intento respetar en todo momento sus decisiones, epidural, acompañante, etc. "(E4M); "Intento sobre todo ser respetuosa con todas las decisiones adoptadas como posibilidad de ver o verlo, su posible autopsia, practica de determinados rituales religiosos, etc." (E29A). Aunque el dolor es inevitable, aunque el profesional no puede traer de vuelta a la vida al neonato (Parkes 1972), los profesionales sienten que sí puede propiciar la despedida; en este sentido los relatos dan especial importancia a este elemento como proceso necesario en la elaboración del duelo: "Animar a ver el feto porque creo que ayuda en el proceso de duelo a despedirse de él" (E30G); "Es necesario que los padres vean a su hijo. Es importante enfrentar para afrontar. Es de las pocas veces que en mi desarrollo profesional aconsejo que hagan algo." (E23M); "Intento que vean a su hijo y les pregunto si quieren estar con él a solas para poder despedirse, lo que creo que ayuda al proceso de duelo" (E12M); "Ofrecerle el bebé a la madre para que lo vea, lo abrace, lo bese y se despida de él." (E6A). Parece que los profesionales lo consideran un elemento vital en la generación del recuerdo: "Estamos aquí para escuchar y ayudar a crear recuerdos para aferrarse y albergar a un bebé que siempre vivirá en su corazón"(E6A). La pérdida es dolorosa, es una de las experiencias más intensas que se pueden vivir y también presenciar (Bowlby 1980), quizás por ello la despedida sea un tema recurrente en los relatos: "Permitir a la pareja que esté el tiempo necesario con su bebé, para despedirse sin presiones y luego aconsejarles que le pongan un nombre y que se despidan de él“" (E34M).

En esta misma línea, otro de los aspectos narrados es la importancia que otorgan los profesionales al fomento de la expresión de los sentimientos, ya que piensan que el duelo debe exteriorizarse precisamente "con signos visibles externos, con comportamientos sociales y ritualidades religiosas" (Pangrazzi 1993: 21): "Es básico intentar crear un clima de confianza en el que la mujer y la familia puedan expresar libremente lo que sienten" (E33M); "El duelo hay que hablarlo, Ilorarlo cuando ellos lo necesiten" (E21M); "Es bueno que exprese sus sentimientos, Ilorando, hablando del tema, hay que saber escucharla“ (E17A). El respeto al llanto y al dolor tamiza casi todos los discursos, desde la consideración de que un buen profesional tiene que tener sensibilidad cuidadora, capacidad de escucha, comprensión, respeto y tolerancia a las expresiones del otro: "Dejarlos tranquilos el tiempo en que lo necesiten, para llorar juntos y que puedan ir asumiendo la pérdida y el do- 
lor" (E2M); "El llanto es el medio que nos da la naturaleza para liberar tensiones, el dolor se puede compartir o mantenerlo en privado, hay que respetarlo $y$ aceptarlo" (E6A). Se trata de acompañarles en silencio: "No me gusta hacerles preguntas, solo me siento cerca y cuidándoles. Cualquier palabra equivocada, puede ser emocionalmente muy dura, así que evito hablar de nimiedades para rellenar los silencios y me muestro cercana mediante comunicación no verbal" (E21M). Desde su posicionamiento el objetivo es transmitirles tranquilidad y apoyo emocional: "Lo único que hay que transmitirles es tranquilidad ya que no podemos hacer nada para remediar lo inevitable, les brindaremos nuestro apoyo emocional" (E31A). Los relatos ponen de manifiesto la importancia de no extrapolar nuestros propios temores (Delgado y Monzón 2002): "No hacer comentarios inapropiados" (E33M). La valía de informar bien al paciente: "Cuidado exquisito de la paciente con información actualizada en cada momento" (E5G); "Hacerlos partícipes de todo el proceso informándolos de los pasos a seguir y que ellos decidan los aspectos que puedan elegir." (E33M); "Tranquilizarla y atender las dudas que le surjan" (E17A). La importancia de saber gestionar su ira y la de sus familiares aunque, a veces, suponga una tarea difícil (Jonas-Simpson et al. 2006): "En ocasiones estos pacientes y sus familiares están enfadados y furiosos, yo procuro escuchar y tener paciencia, tranquilizando a la vez que les dejo sacar su rabia". (E2M); "Hemos de reconducir la ira de manera muy templada y no instigarle ni siquiera de manera sutil“ (E20M). La relevancia de pedir ayuda externa para gestionar ciertos aspectos psicológicos: "Aparte de mi función como matrona en este aspecto veo fundamental la visita de asistencia psicológica a la pareja antes y después del parto por parte del profesional de psicología“ (E22M). Subyace, pues, en los testimonios la necesidad de abordar la muerte perinatal desde una perspectiva holística: "El tema debe ser abordado de forma holística en todas las esferas sobre todo en la psicológica y social, donde las personas involucradas en el proceso tienen que ser apoyadas y tratadas" (E27M). Se observa, en definitiva, la adopción de un abordaje proactivo del duelo propio de la asunción de riesgos del hombre tardo-moderno basado en el fomento de la expresión de los sentimientos, el apoyo emocional y el contacto con el mortinato.

\section{Conclusiones}

En línea con la mayoría de las investigaciones previas, los relatos reflejan las paradojas de unos profesionales de vida que han de enfrentarse a la muerte. En este habitus se dibujan las carencias de una falta de formación institucional, los tics de unos proyectos curriculares que dan prelación a las esferas biológicas, quedando las dimensiones psicosociales al libre albedrío del esfuerzo formativo perso- nal. Desde esta indolencia los expertos desarrollan variopintas estrategias de formación, nutriéndose de fuentes tan diversas como la experiencia profesional o personal, la formación autodidacta, los cursos al margen del itinerario académico oficial, e incluso, si la dificultad apremia, la creatividad, la emotividad o la empatía personal. Surgen, pues, en el discurso las demandas de una mayor implicación institucional en la gestión emocional del duelo y la pérdida fetal.

Alumbrar la muerte es sin duda uno de los momentos más dolorosos para los padres, pero lo es también para los profesionales. No ha de extrañar, pues, que los vivan como situaciones clínicas con tintes de tragedia, como situaciones traumáticas al borde del abismo existencial, como experiencias impactantes que persisten en la memoria. La batalla parece que se juega en el campo de la autogestión emocional, en el ámbito de lo sensitivo, surgiendo una cascada emocional que abarca todo el espectro doloroso de lo sensible: impotencia, incertidumbre, miedo, dolor, tristeza, desolación, ira, culpa, negación, evitación, rechazo, llanto. En concordancia con los estudios previos, los testimonios parecen estar tamizados de dolor ante lo inabarcable de la muerte, estado desde la que los expertos nunca se sienten totalmente preparados para afrontarla, para gestionarla de forma íntegramente satisfactoria en sus pacientes.

Sin embargo, pese al tabú hacia la muerte, pese a las dificultades en la formación y gestión emocional de la pérdida, los discursos muestran un cambio de rumbo respecto a las evidencias mostradas en estudios anteriores; revelan el paulatino asentamiento de un modelo asistencial consecuente con la propuesta de afrontamiento del duelo de Walter, modelo basado en la verbalización de la experiencia, la presencia de la terapia y el contacto con el mortinato. Una perspectiva asistencial desde la que encuentra cabida el cuidado del entorno, la promoción del apoyo sociofamiliar, la mitigación del dolor físico, y sobre todo una gestión psicológica basada en el respeto, en el fomento de la despedida, de la verbalización del dolor y del derecho a la información. Desde esta configuración los testimonios muestran cierta oposición a los modelos de afrontamiento considerados por los informantes como anacrónicos que se centraban en la negación, la evitación de la despedida y la resistencia al contacto materno-fetal tras la muerte.

Se constatan, pues, ciertos cambios en cuanto a la gestión asistencial de la muerte perinatal, ciertas inquietudes hacia lo emocional, hacia el manejo psicológico del duelo, hacia la verbalización de la muerte. Pese a ello, los relatos revelan también una falta de implicación institucional, una deficitaria formación curricular en la materia y una ausencia de guías clínicas basadas en la evidencia. En síntesis, y a tenor de los relatos, se puede concluir que estamos en un momento de tránsito desde configuraciones ideológicas y modelos de afrontamiento del duelo perinatal 
basados en la evitación y el distanciamiento emocional hacia otros basados en la verbalización de la experiencia y el contacto con el mortinato.

\section{Notas}

1. En España la mortalidad perinatal, es decir la que acontece entre las 28 semanas de gestación y los 7 días de vida, asciende a 1729 fallecimientos, de los que 1398 corresponden a muertes fetales tardías acontecidas antes del nacimiento (Instituto Nacional de Estadística 2013).

2. La identificación de los testimonios vendrá dada en adelante por el número de entrevista en el que se participó y

\section{REFERENCIAS BibLIOGRÁficAs}

Allchin, L. 2006. "Caring for the dying: Nursing student perspectives". Journal of Hospice \& Palliative Nursing 8: 112-119. http://dx.doi.org/10.1097/00129191200603000-00015

Bauman, Z. 2001. La posmodernidad y sus descontentos. Madrid: Akal.

Beck, U. 1998. La sociedad del riesgo. Hacia una nueva modernidad. Paidós: Barcelona.

Bertaux D. 1993. "Los relatos de vida en el análisis social". Pp. 136-148 en Historia oral, compilado por J. Aceves. Ciudad de México D.F.: Universidad Autónoma Metropolitana.

Bonanno, G. A. 2009. The other side of sadness: What the new science of bereavement tells us about life after loss. New York: Basic Books

Bowlby, J. 1980. Attachment and loss: Loss, sadness, and depression. Nueva York: Basic Books.

Bourne, S. 1968. "The psychological effects of stillbirths on women and their doctors". British Journal of General Practice 16: 103-112.

Badenhorst, W. and P. Hughes. 2007. "Psychological aspects of perinatal loss". Best Practice \& Research Clinical Obstetrics \& Gynaecology 21: 249-259. http://dx.doi. org/10.1016/j.bpobgyn.2006.11.004

Cacciatore, J. 2010. "Stillbirth: Patient-centered psychosocial care". Clinical Obstetrics Gynecology 53: 691-699. http://dx.doi.org/10.1097/GRF.0b013e3181eba1c6

Cacciatore, J. 2013. "Psychological effects of stillbirth". Seminars in Fetal \& Neonatal Medicine 18: 76-82. http:/l dx.doi.org/10.1016/j.siny.2012.09.001

Camus, A. 1965. Le mythe de Sisyphe. Essais. París: Gallimard.

Charmaz, K. 2006. Constructing Grounded Theory: A Practical Guide through Qualitative Analysis. London: Sage Publications.

Chan, M. F., F. L. Lou, D. G. Arthur, F. L. Cao, L. H. Wu, P. Li, M. Sagara-Rosemeyer and L. Lui. 2008. "Investigating factors associate to nurses' attitudes towards perinatal bereavement care". Journal of Clinical Nursing 17: 509-518. http://dx.doi.org/10.1111/j.13652702.2007.02007.x

Coffey, A. y P. Atkinson. 2005. Encontrar el sentido a los datos cualitativos. Medellín: Universidad de Antioquia de Medellín. las siglas de la profesión a la que se pertenece, "G" para ginecólogos, "M" para matronas y "A" para auxiliares.

3. El término "feto cruz" es utilizado por los profesionales sanitarios para referirse a un 'feto fallecido antes, durante o nada más nacer'.
Côte-Arsenault, D. and E. Denney-Koelsch. 2011. "My baby is a person: Parents experiences with life-threatening fetal diagnosis". Journal of Palliative Medicine 14: 13021308. http://dx.doi.org/10.1089/jpm.2011.0165

Delgado, J. C. \& M. Monzón. 2002. "Estudio sobre la vivencia de la muerte entre los sanitarios". Cultura de los cuidados: Revista de enfermería y humanidades 12: 81-90.

Derrida, J. 1996. Aporías; Morir-esperares (en) los límites de la verdad. Barcelona: Paidós.

Domínguez, B. y S. Urraca. 1985. "Opiniones de los médicos y enfermeras sobre el dolor y la muerte de pacientes terminales". Jano 654: 67-68.

Doka, K. J. 2002. Disenfranchised grief: New directions, challenges, and strategies for practice. Michigan: Research Press Publishers.

Elia, A. D. 1959. "The management of grief situations in obstetrics". BMQ; The Boston medical quarterly 10: 6-12.

Fernández, M., F. Cruz, N. Pérez y H. Robles. 2012. "Factores psicológicos implicados en el duelo perinatal". Index de Enfermería 21: 48-52. http://dx.doi.org/10.4321/ S1132-12962012000100011

Fenwick, J., B. Jennings, J. Downie, J. But y M. Okanaga. 2007. "Providing perinatal loss care: satisfying and dissatisfying aspects form midwives". Women and Birth 20: 153160. http://dx.doi.org/10.1016/j.wombi.2007.09.002

Gabilondo, A. 2003. Mortal de necesidad: la filosofía, la salud y la muerte. Madrid: Abada Editores.

Gálvez, A. 2006. "Muerte alrededor del nacimiento: creencias, sentimientos y vivencias, una perspectiva de los profesionales de los cuidados". Tesis Doctoral. Departamento de Enfermería, Universidad de Alicante, Alicante.

García-Caro, M. P, F. Cruz, J. Schmidt, A. Muñoz, R. Montoya, D. Prados y M. C. Botella. 2010. "Influencia de las emociones en el juicio clínico de los profesionales de la salud a propósito del diagnóstico de enfermedad terminal". International Journal of Clinical and Health Psychology 10:57-73.

Gardner, J. M. 1999. "Perinatal death: uncovering the needs of midwives and nurses and exploring helpful interventions in the United States, England and Japan". Journal of Transcultural Nursing 10: 120-130. http://dx.doi. org/10.1177/104365969901000205

Giddens, A. 1993. Consecuencias de la modernidad. Alianza: Madrid. 
Glaser, B. G. and A. L. Strauss. 1967. The discovery of grounded theory: Strategies for qualitative research. New York: Aldine de Gruyter.

Goffman, E. 1987. La presentación de la persona en la vida cotidiana. Buenos Aires: Amorrortu.

Gold, K. J., V. K. Dalton and T. L. Schwenk. 2007. "Hospital care for parents after perinatal death". Obstetrics and Gynecology 109: 1156-1166. http://dx.doi.org/10.1097/01. AOG.0000259317.55726.df

Gómez, M. 2003. "Morir con dignidad II: La esfera espiritual del hombre al final de la vida. Muerte y religión". Pp. 262-271 en Avances en Cuidados Paliativos. Las Palmas: Gafos.

Hammersley, M. and P. Atkinson. 1994. Etnografía. Métodos de investigación. Barcelona: Paidós Ibérica.

Heazell, A. E. P., M. J. McLaughlin, E. B. Schmidt, P. Cox, V. Flenady, T. Y. Khong and S. Downe. 2012. "A difficult conversation? The views and experiences of parents and professionals on the consent process for perinatal postmortem after stillbirth". BJOG: An international journal of obstetrics \& Gynaecology 119: 987-997. http://dx.doi.org/10.1111/j.1471-0528.2012.03357.x

Heidegger, M. 1997. El ser y el tiempo. Santiago de Chile: Editorial Universitaria.

Hernández, R., C. Fernández y L. Baptista. 2014. Metodología de la investigación. México: Mc Graw Hill.

Instituto Nacional de Estadística. 2013. Secretaría de Estado de Economía y Apoyo a la Empresa. Mortalidad infantil. Tasa de mortalidad perinatal. Madrid: INE.

Jonas-Simpson, C., G. J. Michell, A. Fisher, G. Jones and J. Linscott. 2006. "The experience of being listened to: a qualitative study of older adults in long-term care settings". Journal of Gerontological Nursing 32: 46-53. http://dx.doi.org/10.3928/0098-9134-20060101-15

Jonas-Simpson, C., E. McMahon, J. Watson y L. Andrews. 2010. "Nurses experiences of caring for families whosebabies were born still or died shortly after birth". International Journal for Human Caring 14: 14-21.

Jonas-Simpson, C., F. B. Pilkington, C. MacDonald y E. McMahon. 2013. "Nurses experiences of grieving when there is a perinatal death". SAGE Open 3(2): 1-11. http:// dx.doi.org/10.1177/2158244013486116

Jones, K and L. Smythe. 2015. "The impact on midwives of their first stillbirth". New Zealand College of Midwives Journal 51: 17-22. http://dx.doi.org/10.12784/ nzcomjnl51.2015.3.17-22

Kirkley-Best, E. \& K. R. Kellner. 1982. "The forgotten grief: A review of the psychology of stillbirth". American Journal of Orthopsychiatry 52: 420-429. http://dx.doi. org/10.1111/j.1939-0025.1982.tb01428.x

Kübler-Ross, E. 1969. On death and dying. New York: The Macmillan Company.

Mander, R. 1994. Loss and bereavement in childbearing. Oxford: Blackwell Publishing.

Marín, E. 2007. La muerte en la vida del ser humano. Logroño: Instituto de Estudios Riojanos.

Mejías, M. C, S. García y L. Duarte. 2012. Guía clínica de abordaje del duelo perinatal. Madrid: Bubok Publishing.

(de) Miguel, J. M. 1995. "El último deseo. Para una sociología de la muerte en España”. Revista Española de Investigaciones Sociológicas (REIS) 71-72: 109-156.
Morin, E. 1994. El hombre y la muerte. Barcelona: Editorial Kairós.

Nuzum, D; S. Meaney and K. O'Donoghue. 2014. "The impact of stillbirth on consultant obstetrician gynaecologists: a qualitative study". BJOG: An International Journal of Obstetrics and Gynaecology 121: 1020-1028. http:// dx.doi.org/10.1111/1471-0528.12695

Pangrazzi, A. 1993. La pérdida de un ser querido: un viaje dentro de la vida. Granada: Ed. Paulinas.

Parkes C. M. 1972. Berearement: Studies of grief in adult life. Nueva York: International Universities Press.

Pastor, S. M., J. M. Romero, C. H. Montoro, L. M. Crespo, V. A. G. Jaén and R. M. B. Tirado. 2011. Experiences with perinatal loss from the health professionals perspectives. Latin American Journal of Nursing 19: 1405-1412. http://dx.doi.org/10.1590/S0104-11692011000600018

Pastor, S. M., J. M. Romero, J. C. Paramio, C. Hueso, O. PaIoma, M. Lillo, A. C. Toledano; C. Carnicer; J. A. Ortegón y A. J. Frandsen. 2012. "Tackling perinatal loss, a participatory action research approach: research protocol". Journal of advanced nursing 68: 2578-2585. http://dx.doi.org/10.1111/j.1365-2648.2012.06015.x

Payás, A. 2010. Las tareas del duelo. Psicoterapia del duelo desde un modelo integrativo-relacional. Barcelona: Editorial Paidós.

Pellegrino, E. 1985. "The Caring Ethic: The relation of physican to patient", Pp 8-3 en Caring, curing, coping nurse, physician, patient relationships, editado por A.H. Bishop and J.R: Scudder. Birmingham, AL: University of Alabama Press.

Puia, D. M., L. Lewis y C. T. Beck. 2013. "Experiences of obstetric nurses who are present for a perinatal loss". Journal of Obstetric, Gynecologic, \& Neonatal Nursing 42: 321 331. http://dx.doi.org/10.1111/1552-6909.12040

Roehrs, C., A. Masterson, R. Alles, C. Witt \& P. Rutt. 2008. "Caring for families coping with perinatal loss". Journal of Obstetric, Gynecologic, \& Neonatal Nursing 37: 631-639. http://dx.doi.org/10.1111/j.1552-6909.2008.00290.x

Savage, J. A. 1992. Duelo por las vidas no vividas. Barcelona: Luciérnaga.

Strauss. A. and J. Corbin. 1990. Basics of qualitative research Grounded theory procedures and techniques. Newbury Park: Sage Publications.

Strauss, A. and J. Corbin. 1998. Basics of qualitative research techniques and procedures for developing grounded theory. Thousand Oaks: Sage Publications

Stroebe, M., \& H. Schut. 1999. "The dual process model of coping with bereavement: Rationale and description". Death Studies 23: 197-224. http://dx.doi. org/10.1080/074811899201046

Taylor, S. y R. Bogdan. 1998. Introducción a los métodos cualitativos de investigación la búsqueda de significados. Buenos Aires: Paidós.

Wallbank, S y N. Robertson. 2008. "Midwife and nurse responses to miscarriage, stillbirth and neonatal death: a critical review of qualitative research". Evidence Based Midwifery 6: 100-106.

Walter, T. 1994. The Revival of Death. London: Routledge. http://dx.doi.org/10.4324/9780203220306

Worden, J. W., Á. Aparicio y G. S. Barberán. 1997. El tratamiento del duelo: asesoramiento psicológico y terapia. Barcelona: Paidós. 
JOSÉ MANUEL HERNÁNDEZ GARRE es Doctor en Antropología social y profesor de Historia y Filosofía del Cuidado en la Universidad Católica de Murcia. Es autor de libros como Historia de las matronas. Evolución de la ciencia y el arte de la partería (DM, 2012) o La labor del antropólogo. Reflexiones epistemológicas (DM, 2013) y coautor de diversos artículos en el campo de la antropología de la reproducción como "La parte negada del parto institucionalizado explorando sus bases antropológicas" (RDTP, 2014), "El nacimiento hospitalario e intervencionista: un rito de paso hacia la maternidad" (AIBR, 2015), "Sociología del parto. Conflictos asistenciales en el marco del paradigma tecnocrático" (RIS, 2016), "La catarsis de los cuerpos grávidos. Experiencias de dolor y sufrimiento en el «habitus» del parto medicalizado" (RDTP, 2016), "Hablan las madres. La parte menos contada del aborto voluntario" (Cuadernos de Bioética, 2017) o "¿Némesis obstétrica o disminución del riesgo? A debate bioético el abordaje intervencionista en los partos de bajo riesgo" (Acta bioethica, 2017).

FRANCISCA CARMEN SÁNCHEZ SÁNCHEZ es Diplomada en enfermería especialista en obstetricia y ginecología (matrona). Matrona del Hospital Los Arcos del Mar Menor. Colaboradora externa de la línea de investigación "Antropología de la salud" y del grupo de investigación "Pensamiento en el contexto social" de la Universidad Católica de Murcia. Tras realizar sus estudios de postgrado sobre el duelo perinatal imparte conferencias sobre el mismo a profesionales sanitarios del área de obstetricia de diferentes hospitales del Sistema Nacional de Salud.

PALOMA ECHEVARRÍA PÉREZ es Doctora en Antropología social, Decana de la Facultad de Enfermería, directora del Máster de Investigación en Ciencias Socio-sanitarias e Investigadora Principal del grupo de investigación "Pensamiento en el contexto social" en la Universidad Católica de Murcia. Es coautora de libros como Influencia migratoria en los cuidados informales y la autoatención. Un estudio etnográfico en la ancianidad dependiente (Editorial Academia Española 2012) y de diversos artículos en el campo de la antropología de la salud y de la reproducción como "Análisis antropológico de los patrones de uso y el perfil del usuario de terapias complementrarias orientales" (Gazeta de Antropología 2008), "The cultural dialogue on the domestic dimension of care to immigrant caregivers in Spain" (Rev. Latino-Am. Enfermagem, 2015), "Suffering of Traumatic Amputees in Spain: Meaning, Spirituality, and Biomedicine" (Holistic Nursing Practice 2016) o "Construyendo los roles parentales en el seno de las dinámicas asistenciales institucionales" (RAE, 2016). 